\title{
Geometrical Control of the Interatomic Coulombic Decay Process in Quantum Dots for Infrared Photodetectors
}

\author{
Praphasiri Dolbundalchok, Daniel Peláez; Emad F. Aziz $\stackrel{\text { †nnika Bande }}{\S}$
}

April 25, 2016

\begin{abstract}
In electron dynamics calculations the interatomic Coulombic decay (ICD) process has recently been shown to take place in two vertically-aligned quantum dots (QDs). Energy emitted during the relaxation of one electron in one QD is converted into kinetic energy of another electron ejected from a neighboring QD.

As the electronic structure of QDs can be controlled by their geometries, we prove here in thorough scans of the transversal and vertical QD confinement potentials' widths that geometries are likewise control parameters for ICD. Such a comprehensive investigation has been enabled by a significant development of the calculations in terms of speed achieved among others by optimization of the grid and Coulomb interaction operator representations. As key result of this study we propose two cigarshaped singly-charged GaAs QDs vertically aligned in the direction of their long side for a most efficient QD ICD realization useful for an infrared photodetector.
\end{abstract}

Keywords: Dynamics, Electronic-Structure, Energy Exchange, Model, Systems.

${ }^{*}$ Theoretische Chemie, Physikalisch-Chemisches Institut, Universität Heidelberg, Im Neuenheimer Feld 229, 69120 Heidelberg, Germany

${ }^{\dagger}$ Laboratoire PhLAM - UFR Physique Bâtiment P5, Université Lille 1, 59655 Villeneuve dAscq cedex, France and Theoretische Chemie, Physikalisch-Chemisches Institut, Universität Heidelberg, Im Neuenheimer Feld 229, 69120 Heidelberg, Germany

${ }_{\ddagger}^{\ddagger}$ Institute of Methods for Material Development and Joint Ultrafast Dynamics Lab in Solutions and at Interfaces (JULiq), Helmholtz-Zentrum Berlin für Materialien und Energie GmbH, Albert-Einstein-Str. 15, 12489 Berlin, Germany and Department of Physics, Freie Universität Berlin, Arnimallee 14, 14195 Berlin, Germany

${ }^{\S}$ Institute of Methods for Material Development and Joint Ultrafast Dynamics Lab in Solutions and at Interfaces (JULiq), Helmholtz-Zentrum Berlin für Materialien und Energie GmbH, Albert-Einstein-Str. 15, 12489 Berlin, Germany and Theoretische Chemie, Physikalisch-Chemisches Institut, Universität Heidelberg, Im Neuenheimer Feld 229, 69120 Heidelberg, Germany 


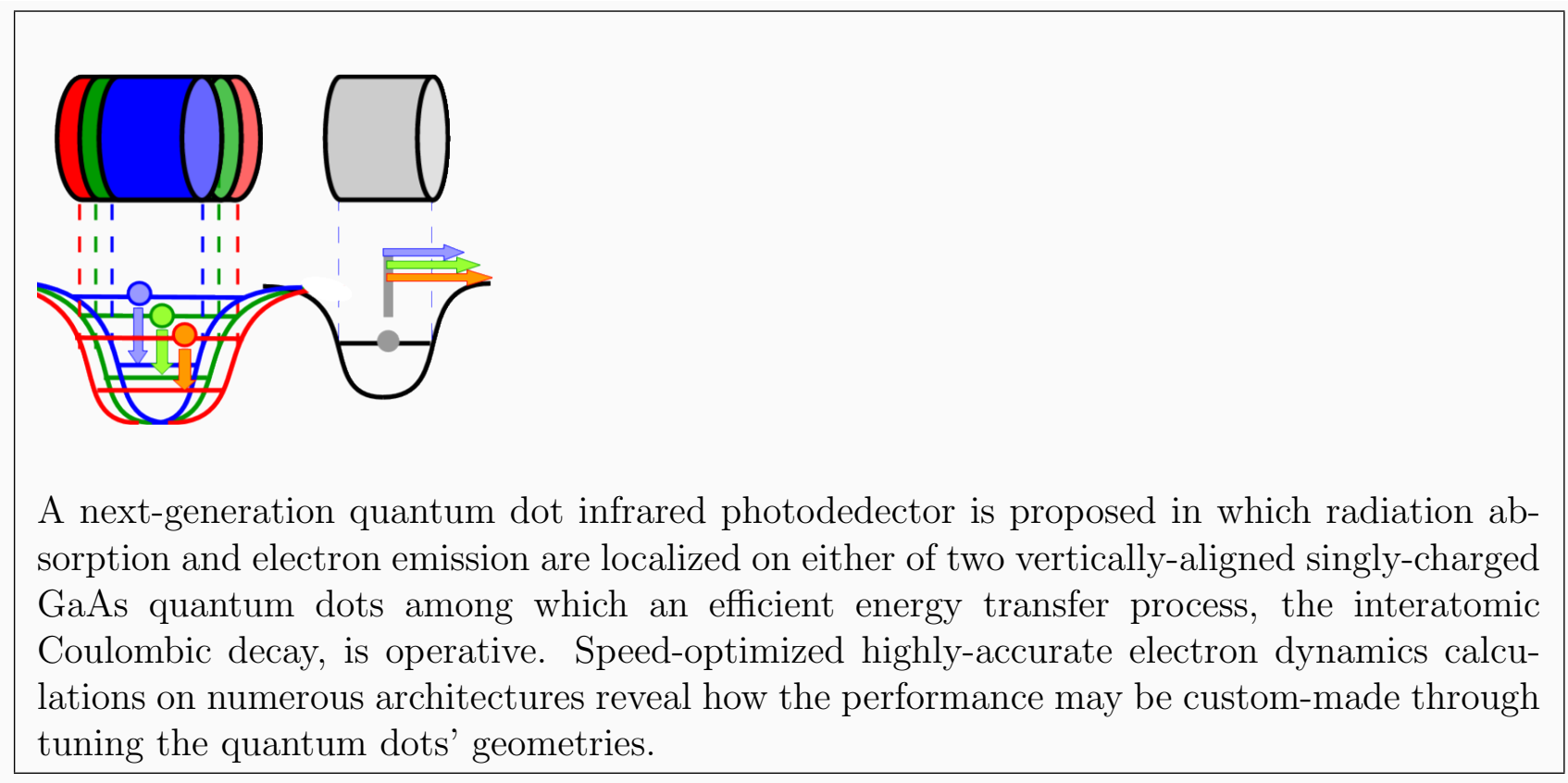




\section{INTRODUCTION}

The ultrafast interatomic (intermolecular) Coulombic decay (ICD) ${ }^{1}$ energy transfer process in which energy from light is converted into an electron current has been investigated theoretically and experimentally for about two decades in molecular systems ${ }^{2-8}$ among which we can find solvated ${ }^{9-13}$ and bio-medical molecules. ${ }^{14-17}$ This decay process is mediated by the long-range Coulomb force of - in the simplest case - only two electrons residing in two electron binding systems (e.g. atoms) neighboring each other, but with a distance long enough to forbid electron transfer. ${ }^{1,18}$ In a general three-level picture the two systems form an array represented by two electron binding potentials (Fig. 1, right side). ${ }^{18}$ Each accommodates one electron, furthermore the left one allows for two of the electronic levels $L_{1}$ and $L_{0}$, the right one for only one level $R_{0}$ as indicated by the arrows in Fig. 1 . The initial state for ICD is a resonance termed $L_{1} R_{0}$ with one electron populating the excited $L_{1}$ level and the other one the $R_{0}$ level. In ICD the $L_{1}$ electron relaxes to the $L_{0}$ level while its emitted energy is transferred to the right binding potential from which the $R_{0}$ electron is excited into a continuum state $C$ according to $\left|L_{1} R_{0}\right\rangle \rightarrow\left|L_{0} C\right\rangle$ when the energy condition $\Delta E_{L}=E_{L_{1}}-E_{L_{0}} \geq I P-E_{R_{0}}=\Delta E_{R}$ for the ionization energy $I P$ of the electron in the $R_{0}$ level is fulfilled. ${ }^{18-20}$

This plain formulation of ICD in a few-level system with a few electrons was the motivation for us to build up expertise in the electron dynamics treatment of ICD and to open up the new field of material science that led both to the prediction of ICD in semiconductor

quantum dots (QDs; or nanocrystals in the chemical sciences ${ }^{21}$ ). ${ }^{18}$ Based on the possibility of designing QDs with certain properties ${ }^{22-25}$ and hence to control processes we propose here the geometric control of the ICD process.

The ICD efficiency for any type of system is represented through the decay rate $\Gamma$ (the inverse of the decay time). To obtain it we employ electron dynamics to solve the timedependent Schrödinger equation and calculate $\Gamma$ from the exponential decay of the squared absolute autocorrelation function

$$
|a(t)|^{2}=\left|\left\langle\Phi_{L_{1} R_{0}}\left(\mathbf{r}_{1}, \mathbf{r}_{2}\right) \mid \Psi\left(\mathbf{r}_{1}, \mathbf{r}_{2}, t\right)\right\rangle\right|^{2}=e^{-\Gamma t}
$$

among the initial resonance state wave function $\Phi_{L_{1} R_{0}}\left(\mathbf{r}_{1}, \mathbf{r}_{2}\right)$ and the time-dependent wave 
function $\Psi\left(\mathbf{r}_{1}, \mathbf{r}_{2}, t\right)$. The calculations are carried out in six dimensions (three spatial coordinates of two electrons) using an antisymmetrized version of the multiconfiguration timedependent Hartree method $\left(\mathrm{MCTDH}^{26,27}\right)$ as implemented in the Heidelberg MCTDH software distribution. ${ }^{28,29}$

In its asymptotic representation $\Gamma \operatorname{reads}^{2,8,30}$

$$
\Gamma=N \cdot \frac{3 c^{2} \hbar^{4}}{4 \pi} \frac{\sigma_{R}^{P I}(\varepsilon) \Gamma_{L}^{r a d}}{E_{v p h}^{4} R^{6}} .
$$

For a given left potential (index $L$ ) $\Gamma$ depends on the amount of virtual photon energy $E_{v p h}=E_{L_{1} R_{0}}-E_{L_{0} R_{0}}$ transferred from the left to the right potential (index $R$ ). Thus it is dependent on the available discrete excited state of the left potential, which also determines the radiative decay rate $\Gamma_{L}^{r a d}$ for $L_{1} \rightarrow L_{0}$. Moreover, $\Gamma$ depends on the photoionzation cross section $\sigma_{R}^{P I}(\varepsilon)$ for a given energy $\varepsilon$ of the electron leaving the right system, which, in turn, depends on $E_{v p h}$. The last influential quantity is the distances $R$ between the excited system $(L)$ and its $N$ neighbors $(R)$.

In an array with the binding systems being atoms or molecules with a given electronic structure there is according to Eq. (2) little room to control the ICD rate. The only option is through $R$ which is, however, barely possible experimentally. In gas phase experiments in which clusters of e.g. 10-100 Ne and Ar or Xe and Ar atoms have been investigated, ensembles of two or three cluster atoms establish a few energetically favored local geometries with $R$ around the van-der-Waals bond lengths. ${ }^{31}$ Similar local geometries are observed in liquid microjet experiments on aqueous clusters. ${ }^{32}$ They are statistically distributed over the complete cluster of the order of nanometers, depending on the monomer concentration, pressure, or temperature. ${ }^{31}$ However, the full control of the cluster composition, and thus of the ICD rate, remains a challenge.

Perfect control of the ICD rate will be possible in solid state QD materials that do not only offer distance control between a clearly defined number of neighbors ${ }^{18,19}$ but more than that a tailor-made electronic structure which allows for control of $\Gamma_{L}^{r a d}, \sigma_{R}^{P I}(\varepsilon)$, and $E_{v p h}$. In semiconductor QDs the conduction band is not continuous as in bulk material, but spatial limitation leads to a size-dependent discretization of the electronic levels known as the quantum size effect. ${ }^{22-24}$ Conclusively, the QD's electronic structure is directly connected to its 
physical size ${ }^{25}$ and maps to the QD representation in terms of a quasi one-dimensional model potential consisting of two inverse Gaussian binding potentials aligned in the energy transfer and electron transport direction and a harmonic oscillator confinement in the transversal directions. ${ }^{18-20}$ We scan systematically through QD geometries and distinguish between the variation of the lateral confinement transversal to the ICD direction (QD width) and the variation of the vertical confinement potential parallel to the ICD direction (QD height) to establish geometric ICD control. For the vertical geometry variation of the left QD we expect a direct impact on the energy levels $L_{0}$ and $L_{1}$ and thus on the energy of the virtual photon $E_{v p h}$ transferred from one QD to the other. According to Eq. (2) this variation is supposed to have most impact on the dynamics as the decay rate in atoms and molecules was predicted to obey $\Gamma \propto E_{v p h}^{-4}$, a fact that we will discuss for QDs. Contrary to this, the lateral geometry variation is not supposed to have its analogue in the rate equation. We aim at investigating if, at all, it does have an effect on the ICD rate and whether we can explain it. In both cases we investigate all QD shapes within the two extremes, cigar and coin.

Note that nowadays several different solid state fabrication techniques of QD arrays exist. In their majority the QDs establish a size anisotropy for the vertical growth direction and the two lateral directions. The prototype for cigar-shaped QDs is a QD in a nanowire that is large in the growth direction and small in the lateral confinement-direction of the wire. ${ }^{33,34}$ QDs in such wires can either be made of a different material than the wire or be gated by electrodes. On the other hand, QDs in an etched pillar structure, are flat objects in growth direction and as wide as the pillar in the other two directions, resulting in coin-shaped structures. ${ }^{35,36}$ Similarly, in self-assembled QDs fabricated by self-organized Stanski-Krastanow growths QDs usually establish laterally wide but flat pyramid or spherical lens shapes that come in vertically-arranged layers forming thus QD arrays. ${ }^{37-40}$

In the theory section of this paper we give at first the details on the QD model and the computations. The results and discussion of the electronic structure and the electron dynamics for the variation of the width and the height of the QDs are then presented in two subsequent sections. To conclude, we point out perspectives for the efficiency-optimized QD array in the device application of ICD in next-generation QD infrared photodetectors.

As previous calculations had turned out to be very time consuming, we present here 
optimized parameters of the program routines, the grid basis, and the representation of operators from which we obtain a significant speedup as detailed in the Appendix. Without this speedup an exhaustive scan of control parameters would not have been possible.

\section{THEORY}

\section{QD Model Hamiltonian}

The two-electron Hamiltonian that we have used in this work reads (in atomic units):

$$
\hat{H}\left(\mathbf{r}_{1}, \mathbf{r}_{2}\right)=\sum_{i=1}^{2} \hat{T}\left(\mathbf{r}_{i}\right)+\sum_{i=1}^{2} V_{E M A}^{e l}\left(\mathbf{r}_{i}\right)+r_{12}^{-1 / 2}
$$

Besides the kinetic energy operators $\hat{T}$ for both electrons and the electron-electron repulsion term $r_{12}^{-1 / 2}=\left|\mathbf{r}_{1}-\mathbf{r}_{2}\right|^{-1 / 2}$ (cf. Computational Details), the Hamiltonian contains the quasi one-dimensional model potential for each electron in all three spatial dimensions which is given according to the effective mass approximation $\left(\mathrm{EMA}^{41}\right)$ in atomic units as: ${ }^{18-20,42-44}$

$$
V_{E M A}^{e l}\left(\mathbf{r}_{i}\right)=0.5 \omega_{\perp}^{2}\left(x_{i}^{2}+y_{i}^{2}\right)-D_{L} \cdot e^{-b_{L}\left(z_{i}-\frac{R}{2}\right)^{2}}-D_{R} \cdot e^{-b_{R}\left(z_{i}+\frac{R}{2}\right)^{2}} .
$$

$V_{E M A}^{e l}\left(\mathbf{r}_{i}\right)$ reflects two QDs vertically-aligned along the $z$ coordinate and being separated by the distance $R=8$ a.u., which corresponds to $86.68 \mathrm{~nm}$ for GaAs QDs (cf. Fig. 1). Note at this point that except for $\omega_{\perp}$ and $b_{L}$ all numerical data in this paper is given in units of GaAs QDs (nm for distances, meV for energies) by using the material specific effective mass and dielectric constant, $m^{*}=0.063$ and $\kappa=12.9{ }^{45}$ and the conversion equations as in previous works. ${ }^{18,46}$ In this vertical $z$ direction the higher energy electrons can be unbound and move freely, the lower-energy electrons are confined by an inverse Gaussian double-well potential for which $D_{L}=1.0$ a.u. $(10.30 \mathrm{meV})$ and $D_{R}=0.8$ a.u. $(8.24 \mathrm{meV})$ indicate the depths of the left and right well, respectively. In the lateral $x$ and $y$ directions the electrons are confined by a harmonic oscillator potential.

Vertical size variation of the QDs can be realized by modifying the parameters $b_{L}$ or $b_{R}$ that determine the full width at half maximum $h_{L, R}=2 \cdot\left(\ln (2) / b_{L, R}\right)^{1 / 2}$ of each potential well. Hence, large values of $b_{L, R}$ represent narrow potentials, i.e. flat coin-shaped QDs while 
small $b_{L, R}$ values represent wide potentials, i.e. high QDs. Throughout this study $b_{R}$ is set to 1.0 a.u. which corresponds to $h_{R}=18.04 \mathrm{~nm}$ in units of GaAs. $b_{L}$ was varied under the conditions that the left potential well binds two electronic levels $L_{0}$ and $L_{1}$ which is in the parameter range $46.59 \mathrm{~nm} \geq h_{L} \geq 29.46 \mathrm{~nm}$. While it is varied, the frequency $\omega_{\perp}$ is kept at a default value of 1 a.u. Using the definition of the diameter of a two-dimensional harmonic oscillator at the frequency of its lowest level ${ }^{47}$ allows us to define the diametral widths of the QDs as $2 r_{\perp}=2 \cdot \sqrt[3]{3 \pi /\left(4 \omega_{\perp}^{2}\right)}$ which is after conversion into units of GaAs $28.84 \mathrm{~nm}$. Hence, height varied QDs range from approximately spherical to short cigar-shaped QDs.

We also vary the width of the QDs as $133.85 \mathrm{~nm} \geq 2 r_{\perp} \geq 6.21 \mathrm{~nm}$ for a constant height $h_{L}=36.08 \mathrm{~nm}$. These lateral variations are more considerable than the height variation, ranging from the extreme of wide coin-shaped QDs to cigar-shaped QDs, as there are no limitations on the number of bound electronic levels in this case.

\section{Computational Details}

In MCTDH the wave function

$$
\Psi\left(\mathbf{r}_{1}, \mathbf{r}_{2}, t\right)=\sum_{i} \sum_{j} A_{i j}(t) \varphi_{i}\left(\mathbf{r}_{1}, t\right) \varphi_{j}\left(\mathbf{r}_{2}, t\right)
$$

is propagated in time. Generally we assume both electrons in a triplet state by imposing antisymmetry through $A_{i j}=-A_{j i}$. A singlet configuration has been already discussed elsewhere. ${ }^{19}$ It is noted that we have time-dependent orbitals which in the MCTDH community are known as single-particle functions (SPFs) $\varphi_{i}$.

After the preparation of the geometrically varied double QD potentials any electron dynamics calculation is performed in a three-step procedure consisting of initial state preparation, time propagation of the wave function, and analysis of the time-dependent wave function and other observables.

The first step is the determination of an initial time-independent wave function by solving the time-dependent Schrödinger equation for negative imaginary times (i.e. relaxation) yielding a set of 52 triplet eigenstates $\Phi_{n}\left(\mathbf{r}_{1}, \mathbf{r}_{2}\right)$ and their corresponding eigenenergies $E_{n}$. In particular, we have used the block improved relaxation algorithm. ${ }^{29}$ Among all of the 
states, the $L_{1} R_{0}$ resonance and the $L_{0} R_{0}$ ground state can be identified as localized states since they display most of the electron density in the spatial area of the two QDs as was deduced by inspecting the electron density $\rho\left(z_{1}\right)=\int\left|\Psi\left(\mathbf{r}_{1}, \mathbf{r}_{2}, 0\right)\right|^{2} d \mathbf{r}_{2} d x_{1} d y_{1}$ along the $z_{1}$ coordinate for one of the electrons.

The central second step is the actual propagation of the wave function according to the electronic time-dependent Schrödinger equation, with SPFs and coefficient vectors being now propagated in real time. In most cases the propagations are performed using the resonance state $L_{1} R_{0}$ as the initial state. Only in the weakest confinement regime (large $2 r_{\perp}$ ) where $L_{1} R_{0}$ is not within the lowest states obtained in the block improved relaxation, we choose $L_{0} R_{0}$ as initial state and analyze ICD after laser excitation, which can be done as shown in another paper by one of the authors. ${ }^{20}$ After technical improvements of MCTDH parameters for stable, accurate, and fast numerical calculations (cf. Appendix), we choose an integrator accuracy for SPF and A vector propagation of $I \approx 10^{-6} \mathrm{meV}$ and we utilize eight SPFs to represent the wave function. The complex absorbing potentials (CAPs) $W_{z}^{L}$ and $W_{z}^{R}$ on the left or the right side of the double-well of the following form are employed

$$
-i\left(W_{z}^{L}+W_{z}^{R}\right) \text { with } W_{z}^{L / R}=\eta\left|z-z_{0}^{L / R}\right|^{n} \Theta\left(z-z_{0}^{L / R}\right)
$$

to absorb the emitted ICD electrons at the grid's boundaries. Their order is $n=4$ and their onset is determined by the Heaviside step function $\Theta\left(z-z_{0}^{L / R}\right)$ at $z_{0}^{L / R}= \pm 325 \mathrm{~nm}$ resulting in a CAP width of $w=217 \mathrm{~nm}$. The strength is adjusted to $\eta=8.6997 \cdot 10^{-6}$ to obtain optimal absorption properties for the electron. Finally we determine the ICD rate $\Gamma$ from the exponential decay of the squared absolute autocorrelation function (Eq. (1)).

In MCTDH operators and wave functions are represented on a DVR grid. Here identical grids with $N_{i}$ grid points are used for both indistinguishable electrons along each of the coordinates $i=x, y$ or $z$. In the continuum $z$ coordinate a sine DVR is utilized, in the lateral coordinates a harmonic oscillator DVR serves the representation of the bound state in the harmonic QD confinement. The grid optimized for and used throughout this study is $N_{z-} N_{x-} N_{y}=185 \_5 \_5$ compared to the much larger 320_8_8 grid of earlier works. ${ }^{18-20}$ It spans an interval of $[-\mathrm{L} ; \mathrm{L}]=[-542 \mathrm{~nm} ; 542 \mathrm{~nm}]$ rather than $[-1084 \mathrm{~nm} ; 1084 \mathrm{~nm}]$ along the $z$ coordinate. In $x$ and $y$ the representation of the confinement is now defined through its 
analytical form rather than through interpolation (see Appendix).

For the evaluation of the six-dimensional Coulomb integrals at each time step of the electron dynamics calculation compared to earlier calculations of QD ICD ${ }^{18-20}$ and of the related QD interatomic Coulombic electron capture process, ${ }^{42-44}$ we use the POTFIT algorithm to transform the regularized Coulomb potential (Eq. (3)) into a sum of products of one-dimensional single-particle potentials $(\mathrm{SPPs}) v^{(\kappa)}$ :

$$
V^{P F}{ }_{i_{1}, \ldots, i_{f}}=\sum_{j_{1}=1}^{m_{1}} \ldots \sum_{j_{f}=1}^{m_{f}} C_{j_{1}, \ldots, j_{f}} v_{i_{1}, j_{1}}^{(1)} \ldots v_{i_{f}, j_{f}}^{(f)}
$$

where the index $i$ runs along the grid and the index $j$ along the SPP basis functions. Here we step back from the exact expansion with $m_{\kappa}=N_{\kappa}$ along the total number of grid points, but reduce $m_{\kappa}$ for potentially faster calculations at a desired accuracy. Further speedup is achieved by limiting the maximal value of the potential to a threshold of vcut $=10.3 \mathrm{meV}$ (1 a.u.) These two technical improvements have led to propagations being 20 times faster than previous ones and having the same accuracy (see Appendix).

\section{RESULTS AND DISCUSSION}

\section{Control of ICD by QD Width Variation}

\section{Electronic Structure}

In our quasi one-dimensional model for two vertically-stacked QDs the diameter of both

cylindrically-shaped QDs shown in Fig. 1 is varied in the range $84.32 \mathrm{~nm} \geq 2 r_{\perp} \geq 6.21$ $\mathrm{nm}$ which corresponds to the range of confinement strengths 0.2 a.u. $\leq \omega_{\perp} \leq 10.0$ a.u. of the harmonic oscillator potential in Eq. (4). By keeping the heights of the dots constant at $h_{L}=36.08 \mathrm{~nm}$ and $h_{R}=18.04 \mathrm{~nm}$, respectively, we cover a range of geometries from cigar-shaped strong confinement QDs to coin-shaped weak confinement QDs as schematically depicted on the left and right side of Figs. 2 (a) and (b) for the confinement potential and the QD's geometric shape.

Fig. 2 (c) and (d) show the dependence of the resonance and ground state energies $E_{L_{1} R_{0}}$ 
and $E_{L_{0} R_{0}}$ on the the diameter $2 r_{\perp}$ which were obtained from block relaxation calculations on the grid system 185_5_5 with the Coulomb potential constructed from 4300 SPPs. These energies were shifted by setting the energy of the first continuum state $E_{L_{0} C}=0$ meV which has a negligible Coulomb interaction energy contribution because electrons are on average located far apart from each other.

For the energy-to-width relation of the two local two-electron states $L_{0} R_{0}$ and $L_{1} R_{0}$ in Fig. 2 (c) and (d) a weak and a strong confinement regime can be distinguished in which the slopes of the energy curves are different. For the case of a strong transversal confinement with $2 r_{\perp}<28.84 \mathrm{~nm}$ the energies increase in a below-linear manner and for both states eventually converge to a saturation value at $2 r_{\perp} \rightarrow 0$. This effect can be rationalized by considering the electron probability density along $x$ and $y$ as depicted schematically in Fig. 2 (a). As the QDs become narrower the electron in each QD becomes more and more localized along the connection axis through the center of both QDs and the effective electron separation $r_{12}^{\text {eff }}$ converges towards a nominal distance $R \leq 81.7 \mathrm{~nm}$ for $2 r_{\perp} \leq 6.21 \mathrm{~nm}$ and a corresponding classical estimante of the Coulomb interaction is $1 / R=1.367 \mathrm{a.u}$. (see Tab. 1 ). At the same time the relative position of the Gaussian potential energy levels $E_{L_{0}}, E_{R_{0}}$, and $E_{L_{1}}$ remains unaffected by the variation of $2 r_{\perp}$.

Inversely, in the weak confinement regime of $2 r_{\perp} \geq 28.84 \mathrm{~nm}$ the energies decrease linearly upon increasing $2 r_{\perp}$. This can again be understood from the lateral electron density of the broad harmonic oscillator in $x$ and $y$ direction which itself is wide (Fig. 2 (a), left panel). As a consequence the electrons on neighboring vertically stacked QDs separated by the distance $R$ in the $z$ direction can avoid each other efficiently by establishing an effective longer distance of $r_{12}^{e f f}>R$ among them as schematically illustrated in Fig. 2 (b). Hence, the effective Coulomb interaction $1 / r_{12}^{\text {eff }}$ between the electrons is considerably smaller than $1 / R$ and so is the total energy of the localized states. For an infinitely wide harmonic oscillator confinement, which means a stacking of two quantum films rather than quantum dots, we would expect the two-electron state energies to drop down to the respective energy without Coulomb interaction as was analyzed in our first paper of this series. ${ }^{18}$ For an example case of $2 r_{\perp}=2884 \mathrm{~nm}$ we do, however, still obtain for the $L_{0} R_{0}$ state a Coulomb energy of $E_{C}=0.142$ a.u. which connects to $r_{12}^{\text {eff }}=785 \mathrm{~nm}$. 
One last aspect regarding the weak limit of the transversal confinement $2 r_{\perp}$ is that higher excited states in $x$ and $y$ come into play within the energy interval of about $10-20 \mathrm{meV}$ relevant for ICD. However, due to symmetry reasons these states are not occupied during the ICD process and thus they do not influence the physics here. They only have an impact on the states obtained in the block relaxation calculation: when in the weak confinement regime a large number of such states with energies separated only by very small energy differences appears then the calculation starts to suffer from convergence failures. Theses are particularly severe for the resonance state which, for small $2 r_{\perp}$, is no longer among the first 20-30 states but far above.

\section{Electron Dynamics}

In this part we present the results for the electron dynamics calculations of the ICD process under variation of the widths of the QDs defined through their transversal confinement potential $V(x, y)$. These propagations start from an $L_{1} R_{0}$ resonance wave function which evolves in time as can be seen from the exponential decay of the squared autocorrelation function (Eq. (1)) shown in a logarithmic representation in Fig. 3 for the system of our previous studies $\left(2 r_{\perp}=28.84 \mathrm{~nm}\right.$, solid line, default $)$ and the smallest and highest $2 r_{\perp}$ of the present investigations (dashed lines). Note that at small times the decay is either parabolic or faster than exponential due to equilibration and therefore not shown. The figure reveals that for the largest $2 r_{\perp}$ the decay is slowest and then it is basically identical for the default and for smaller $2 r_{\perp}$ although the related harmonic oscillator frequency $\omega_{\perp}$ does not have a direct impact on any of the quantities in Eq. (2). This key result, the decay rate $\Gamma$ as function of the QD widths $2 r_{\perp}$, is presented in Fig. 2 (e) and Tab. 1.

In the weak confinement regime of $2 r_{\perp} \geq 28.84 \mathrm{~nm}$ in which the width of the verticallyarranged QDs becomes larger than their height, the decay rates $\Gamma$ decrease strongly with increasing $2 r_{\perp}$ values. This behavior is parallel to the drop of the resonance state energy $E_{L_{1} R_{0}}$ seen in Fig. $2(\mathrm{~d})$ and of the Coulomb energy $E_{C}$ listed in Tab. 1. Such a behavior was to be expected: ICD is a process driven by the Coulomb interaction and in the case of a small one, state energies and decay rates are likewise small, as we have reported in our prior work in which these quantities were investigated as functions of $R .{ }^{18}$ The rationalization for 
the lowered Coulomb interaction has been given in the previous section through discussing $r_{12}^{e f f}>R$. Consequently ICD in this $2 r_{\perp}$ region slows down as can be deduced from Eq. (2) if $R$ is replaced by $r_{12}^{e f f}$.

In the strong confinement regime of $2 r_{\perp}<28.84 \mathrm{~nm}$, the decay rate initially drops slightly and is then basically independent of $2 r_{\perp}$ (see Fig. 2 (e)). The effective Coulomb potential $1 / r_{12}^{\text {eff }}$ converges for smaller $2 r_{\perp}$ giving a constant ICD rate. For the peak of $\Gamma$ around $2 r_{\perp}=18.17 \mathrm{~nm}$ no self-contained explanation is at hand as neither the Coulomb energy $E_{C}$ has a maximum nor the virtual photon energy $E_{v p h}$ a minimum in that range (Tab. 1). Conclusively, we can say that in all QDs that are either spherical or elongated in binding direction $z$ the ICD rate is of similar value as in our previous study. ${ }^{18}$ A further increase of $\Gamma$ through variation of $2 r_{\perp}$ cannot be expected.

\section{Control of ICD by QD Height Variation}

\section{Electronic Structure}

Another alternative to affect the electronic structure of our quasi one-dimensional model for two vertically-stacked QDs is height variation of the left cylindrically-shaped QD by $46.59 \mathrm{~nm} \geq h_{L} \geq 28.53 \mathrm{~nm}$ through the Gaussian confinement potential parameter 0.15 a.u. $\leq b_{L} \leq 0.40$ a.u. of Fig. 1 and Eq. (4). The range of the parameter $h_{L}$ was selected such that there are always two one-electron levels in the left potential well. Figs. 4 (a), (b) and Tab. 2 show that large $h_{L}$ values result in wide inverse Gaussian potentials reflecting high, cigar-shaped QDs (left panel) whereas small $h_{L}$ values render narrow Gaussian potentials representing coin-shaped QDs (right panel).

The energies of the $L_{0} R_{0}$ ground and the $L_{1} R_{0}$ resonance state as functions of $h_{L}$, relative to the energy of the first continuum state $L_{0} C$ are shown in Figs. 4 (d) and (c), respectively. The ground state energies are basically constant. They only grow by $0.004 \mathrm{meV}$ with decreasing $h_{L}$ and then drop by $0.001 \mathrm{meV}$ for $h_{L} \leq 36.08 \mathrm{~nm}$. The reason is that a decrease of $h_{L}$ leads to an increase of the one-electron level $L_{0}$ and with that to an increase of the unshifted $E_{L_{0} R_{0}}$ by the same amount as the increase of $E_{L_{0} C}$. 
Compared to the marginal changes of $E_{L_{0} R_{0}}$ the increase of the $L_{1} R_{0}$ state energy by $1.214 \mathrm{meV}$ in that respective $h_{L}$ range is considerable. The resonance state raises in energy with decreasing $h_{L}$ mainly because of the narrowing of the Gaussian well that pushes up the $L_{1}$ level considerably more than the $L_{0}$ level. At the same time the Coulomb interaction decreases, but only by $0.051 \mathrm{meV}$, leading to a slight increase of the effective distance $r_{12}^{\text {eff }}$ between both electrons by $3.25 \mathrm{~nm}$ The interpretation is that electrons in higher and spatially more delocalized states can avoid each other more effectively then electrons confined more strongly.

\section{Electron Dynamics}

In this section we present the impact of the $h_{L}$ variation on the dynamics of two confined electrons in the double QD model potential. The decay is displayed in Fig. 3 as dotted lines. Inspection shows that ICD is more sensitive to $h_{L}$ rather than $2 r_{\perp}$ variation and that it is fastest for large $h_{L}$ and slowest for small $h_{L} . \Gamma\left(h_{L}\right)$ is given in Tab. 2 and Fig. 4 (e). It is apparent that the decay rate $\Gamma$ almost linearly decreases with increasing $h_{L}$. This might appear counter-intuitive because $E_{L_{1} R_{0}}$ increases at the same time (Fig. 4 (c)) and earlier we always had connected large $E_{L_{1} R_{0}}$ with large $\Gamma$ (see previous section and literature ${ }^{18}$ ). However, $\Gamma$ depends on the Coulomb energy $E_{C}$ (Tab. 2) which is indeed larger for large $h_{L}$ and vice versa. We further observe that as $E_{C}$ changes by $0.05 \mathrm{meV}$. $\Gamma$ changes by less than one order of magnitude, whereas in our previous studies the respective change of $\Gamma$ was found to be more dramatic, with variations ranging over four orders of magnitude upon a change of $E_{C}$ by $0.72 \mathrm{meV} .{ }^{18}$ Both observations give us a first hint that $\Gamma$ is influenced quite strongly by other quantities rather than by the Coulomb energy $E_{C}$.

The most obvious candidate for such quantity is the virtual photon energy $E_{v p h}$ which increases with decreasing $h_{L}$ as shown in Tab. 2. According to Eq. (2) it would connect to the decay rate as $\Gamma \propto E_{v p h}^{-4}$ if all other quantities in that equation were not affected by a change in $h_{L}$. The respective graph is visualized in a doubly logarithmic representation in Fig. 4 (f). It shows a monotonic decay of $\Gamma$ with increasing $E_{v p h}$ which is first smooth and linear with a slope of -1.69 for $E_{v p h} \leq 5.02 \mathrm{meV}$. Then it strongly drops and continues to decrease linearly for $E_{v p h}>5.40 \mathrm{meV}$ now with a slope of -18.17 . The slopes are in the 
order of magnitude of the anticipated value of -4 . Their deviation indicates a more complex dependence of $\Gamma$ on $h_{L}$.

Upon closer inspection two other quantities in Eq. (2), namely the photoionization cross section $\sigma_{R}^{P I}$ for an electron in the right potential well and the radiative decay rate $\Gamma_{L}^{r a d}$ of the excited state of the $L_{1}$ electron into the ground state, depend themselves on the energy of the virtual photon. We calculated an estimate of both quantities based on single-electron wave functions obtained in this work. The photoionization cross section was obtained according to

$$
\sigma_{R}^{P I}=\frac{4 \pi^{2}}{3 c} \cdot E_{v p h} \cdot\left|\frac{\left\langle\phi_{v p h}|\mu| \phi_{R_{0}}\right\rangle}{\sqrt{\Delta_{\varepsilon}}}\right|^{2}
$$

in which the continuum wave function $\phi_{v p h}$ belongs to the state approximately at the energy $E_{v p h}+E_{R_{0}} \cdot{ }^{20,48}$ The continuum has been discretized as in earlier works ${ }^{20}$ with a density of continuum states given through $\Delta_{\varepsilon} \cdot \sigma_{R}^{P I}$ is in the order of magnitude of 0.1 a.u. and monotonically decreases with $b_{L}$ more quickly than $\Gamma$.

The radiative decay rate was estimated according to ${ }^{18}$

$$
\Gamma_{L}^{r a d}=\frac{4 E_{v p h}^{3}}{3 c^{3}}\left|\left\langle\phi_{L_{1}}|\mu| \phi_{L_{0}}\right\rangle\right|^{2}
$$

It turns out to be in the order of $10^{-8}$ a.u. and to monotonically increase with $b_{L}$ as a square root function. If we considered $\Gamma /\left(\sigma_{R}^{P I} \cdot \Gamma_{L}^{r a d}\right) \propto E_{v p h}$ in a figure as Fig. 4 (f) the graph $\log \left(\Gamma /\left(\sigma_{R}^{P I} \cdot \Gamma_{L}^{r a d}\right)\right) \propto X \log \left(E_{v p h}\right)$ would display unsystematic oscillations rather than a linear slope of -4 . Partly this goes back to the less accurate calculation of the integrals in Eqs. (8) and (9) compared to the determination of $\Gamma$ from Eq. (1). Hence, based on observations we come to the conclusion that $\Gamma$ depends on $b_{L}$ in a non-trivial way as most influential quantities, i.e. $E_{L_{1} R_{0}}, E_{v p h}, \Gamma_{L}^{r a d}, \sigma_{R}^{P I}$, and $E_{C}$, depend on $b_{L}$. There is, however, a qualitative argumentation that we can offer which connects to a geometric argument.

The effect that varying the left potential well along the $z$ coordinate has on the decay rate can be interpreted qualitatively by looking at the illustration of the double QD. In Figs. 4 (a) and (b) the distance $R=86.68 \mathrm{~nm}$ between the minima of the two potential wells representing the centers of mass of the QDs is identical for all $h_{L}$. In contrast to the representations of the model system for different $2 r_{\perp}$ in Fig. 2 (a) and (b) the effective distances $r_{12}^{e f f}$ between confined electrons are not expected to differ much as $h_{L}$ changes. 
However, the spacing $r$ between the edges of two vertically-arranged QDs increases with

decreasing $h_{L}$ and is a boundary for $r_{12}^{e f f}$. Consequently, the energy transfer becomes slower with increasing $r$. In QD materials a spacing $r$ would represent the thickness of a wetting layer or a barrier between QDs. ${ }^{49,50}$ Hence, ICD will be fastest through a thin barrier.

\section{Perspectives for Infrared Detection}

The possibility to custom-make QD properties by geometry control is the key to the QD's success in device applications. For QD ICD in general, where radiation induces and electron current, we envision application of ICD in energy conversion technology. For the infrared light-induced ${ }^{20}$ conduction-band ICD process discussed here this is a next-generation QD infrared photodetector. Current QD infrared photodetectors are typically lens-shaped self-assembled QDs vertically grown in layers. ${ }^{51}$ As they have many bound levels in the transversal dimensions that supress ionization, detection is only in the growth direction. Early detectors used to have several bound states in that direction as well, leading to a relatively inefficient infrared detection as the few accessible bound-to-continuum transitions inducing a photocurrent (where the electrons are emitted into the surrounding medium) are less probable than the bound-to-bound transitions. State-of-art detectors have been further miniaturized such that their height is small enough to allow only for a single electronic level in growth direction. ${ }^{51,52}$ Such photodetectors operate already more efficiently due to the lack of an alternative excitation pathway for electrons from this single level ${ }^{52,53}$ despite the low transition dipole moment of this bound-to-continuum state transition itself. In QD ICD infrared light is temporarily exciting a conduction band electron in the left QD from $L_{0} \rightarrow L_{1}$ in an efficient bound-to-bound transition followed by the likewise efficient ICD process where an electron current from the second QD is induced. ${ }^{20}$ This will again lead to an enhancement of the IR photodetector efficiency, which is the case for all QD geometries studied, i.e. also for the typical flat-and-wide infrared detector QD geometry. However, here we show that QD ICD is significantly more efficient in cigar-shaped vertically-aligned QDs, hence we propose a detector geometry different from the one commonly used. 


\section{CONCLUSIONS}

In summary, we have performed electron dynamics calculations with MCTDH in quasi onedimensional model potentials representing QDs. Compared to earlier calculations we have been able to reduce the computation time by a factor of 20 mainly through improvements of the grid representation and approximation of the Coulomb interaction potential. This development will be of significant importance as we envision the QD ICD process in all possible types of QD arrays as laterally-coupled $\mathrm{QDs}^{54,55}$ or colloidal $\mathrm{QDs}^{23,24,56}$ in which the electronic continuum spans over two or even three spatial dimensions.

Furthermore, we have demonstrated how ICD is controlled by the QD's geometries. On the one hand we varied the widths of both QDs at constant heights going from flat objects as known for etched QDs all the way to cigar-shaped QDs as typically encountered in nanowires. The decay times were 269 ps and 163 ps, respectively, for GaAs QDs. On the other hand a variation of the height of the excited QD which is leading to the same frontier geometries, however with different overall sizes and effective electron-electron distances, gave decay times of 434 ps for the flatter and 116 ps for the higher, more cigar-like QD. We showed elsewhere that phonon-mediated decay is not competitive to ICD with this speed. ${ }^{20}$

Finally, the optimal double QD's geometry that we would like to propose for a first experiment on QD ICD to be realized in charged GaAs QDs turns out to have two aligned cigar-shaped QDs in shortest possible distance from each other. At a separation of $87 \mathrm{~nm}$ optimal diameters are $36 \mathrm{~nm}$. The electron emitting QD shall be $18 \mathrm{~nm}$ high, the other one $36 \mathrm{~nm}$ and the decay time will consequently be $116 \mathrm{ps}$. Such process initiated by IR light absorption ${ }^{20}$ may be ground breaking on the way to a device application in infrared photodetectors.

\section{ACKNOWLEDGMENTS}

We would like to acknowledge financial support by Heidelberg University through the OlympiaMorata fellowship and a grant from the Forschungspool. In addition to that A. B. is grateful to the Deutsche Forschungsgemeinschaft (BA 3770) as well as to the VolkswagenStiftung (Freigeist fellowship 89525). P. D. acknowledges her student fellowship by the Thai govern- 
ment. The authors thank Lorenz S. Cederbaum and Jochen Schirmer for the revision of this manuscript.

\section{APPENDIX}

The speedup of MCTDH two-electron calculations on the ICD process in QD arrays sets on from the quasi one-dimensional QDs of earlier works ${ }^{18-20}$ in which systematic calculations would have been forbiddingly time-consuming and foreseeable calculations with a two- or three-dimensional continuum completely undoable. We optimize the $z$ grid through the grid size, the CAPs, and the Coulomb integral evaluation for a reduced number of single-particle potentials (SPPs) and with cutting the Coulomb potential at the coincidence point of both electrons firstly for the true one-dimensional and then for the full quasi one-dimensional calculations.

The detailed results of the speedup optimization with comparable accuracy are summarized in Tabs. 3 through 5 with subsequent line numbering. As a measure of accuracy we consider the ICD rates $\Gamma$ which are sensitive quantities in the range of a few thousands of a $\mathrm{meV}$. We furthermore present the deviation $\Delta \Gamma$ in percentage terms from the reference value of $\Gamma^{\text {Ref }}=4.1302 \cdot 10^{-3} \mathrm{meV}$ obtained from one of our best-converged calculations (line 32 in Tab. 5). Finally, the real CPU time $T_{\text {Prop }}$ on a single compute node is given for a propagation time of $12.8 \mathrm{ps}$ in units of GaAs. $T_{\text {Prop }}$ is to be understood as indicating a general trend for the calculations, because it depends to a limited extend on the performance of the specific processor and the work load of the compute cluster used for each individual calculation.

In Tabs. 3 and 4 we focus on the grid in $z$ direction and therefore analyze calculations for an effective one-dimensional system instead of the full quasi one-dimensional system. The applicability of this grid reduction for testing purposes in a well-converged grid has extensively been discussed. ${ }^{42,44}$ Note that the correspondence of quasi one-dimensional and one-dimensional grids is perfect in the case of a fully-optimized parametrization (lines 3 and 28 (Tab. 5). Here, lines 27 and 1 give an example for a case, where an imperfect correspondence causes a deviation of both $\Gamma$ from each other which relates to only a limited accuracy 
of the representation of the harmonic oscillator DVR in $x, y$ direction on the quasi onedimensional grid by interpolation of eight grid points. It also comes from the low integrator accuracy of $I=10^{-6}$ a.u. $\left(10^{-5} \mathrm{meV}\right)$.

The increase of integrator accuracy in lines 2-4 shows the convergence of $\Delta \Gamma$. For larger and smaller grids (lines $3 \& 4$ and $7 \& 8$, respectively) the stability of calculations with the integrator accuracy $I=10^{-6} \mathrm{meV}$ is shown together with the savings in computation times which motivates the use of this integrator throughout this work. Moreover, in lines 3 through 8 the transition from a larger to a smaller grid i.e. from 320 points on $[-L ; L]=$ [-1084 nm; $1084 \mathrm{~nm}$ ] to 224 points on [ $-867 \mathrm{~nm} ; 867 \mathrm{~nm}]$ with similar spacings between grid points $\left(d_{z}=N_{z} / 2 L=0.129-0.148\right)$ is shown. The $N_{z}=224$ grid is then used to make further improvements as e.g. shown in lines 6 and 7 where we demonstrate that eight SPFs instead of ten are sufficient for the convergence of $\Gamma$. In lines 9-11 we show for a grid of $L=1625 \mathrm{~nm}$ that the grid density $d_{z}$ should be above 0.111 to maintain accuracy. The subsequent reduction of the grid then conserves $d_{z}=0.129$ in lines $5-8,12-14$. The data in line 14 shows that the reduction of the grid length has a lower limit for $L=433 \mathrm{~nm}$. This is well understandable, because the wider of the two QDs has a spatial extension of at least $108 \mathrm{~nm}$, each CAP needs $217 \mathrm{~nm}$ thus only $108 \mathrm{~nm}$ of space for the continuum electron is left. As an intermediate result the grid with $L=542 \mathrm{~nm}$ and $N_{z}=140$ appears, in terms of timing and accuracy, desirable. As a further aspect of the grid we tested (lines 15 and 16) whether a change from the sine to an exponential DVR would bring a gain in computational effort and turned out not to be the case. The complex absorbing potentials need as well some careful adjustment. A well-behaved CAP would not influence the decay rates other than numerically as do all CAPs of different orders $n=2,3,4$ shown in lines 13, 17, 18 and of different widths $w=217,325,433 \mathrm{~nm}$ in lines 12, 15, 19. As all choices of CAPs give similar accuracy and timing. Thus we finally use a CAP with $n=4$ and $w=217 \mathrm{~nm}$.

The next step in the reduction of numerical effort, from which we expect most savings, is the approximation in the expression of the Coulomb potential by using the POTFIT algorithm. ${ }^{28,57,58}$ An initial major test is made with the one-dimensional system $\left(N_{z}=140\right.$, line 13). We find that a reduction of the number of SPPs $\left(m_{\kappa}\right)$ leads to a low accuracy potential expansion. As we notice, most of the eigenvalues (natural weights) in the expansion 
are large, reflecting a somewhat sparse grid. Hence, a reduction of the number of SPPs should lead to a decrease in computation time at the prize of a decrease in the quality of the potential and, in turn, to a worsening of the quality of our observable $\Delta \Gamma$ which is not significant for $m_{\kappa}=130$ (line 20) but very severe for $m_{\kappa}=80$ (line 21). Counter-intuitively, computation time increases due to the overlay with a second effect, the computation accuracy.

At this point, it is noticed that the used primitive grid leads to far too energetic regions of the potential which are not sampled by the wave function. Hence, we applied a cut on the potential near the coalescence point of both electrons, i.e. for $r_{12} \rightarrow 0$, to a maximum value of $10.30 \mathrm{meV}$ as the ICD electrons never get close together (line 22). Moreover, such an energy cut exceeds the Coulomb energy contribution to the total energies. Instead, with a lower energy cut of $5.16 \mathrm{meV}$ considerable deviations of $\Gamma$ were observed (line 23). With the Coulomb potential truncated at $10.30 \mathrm{meV}$ we turn to a slightly larger grid of 185 points again (lines 24-26) for which the reduction of SPPs did then give the anticipated improvement on the computation time without a loss of accuracy for $\Gamma$. It should be remarked that we envisage the use of the new Multigrid POTFIT method ${ }^{59}$ to deal with the issues associated with the increase in the number of dimensions or electrons, i.e. the grid size.

Finally, we turn to the quasi one-dimensional two-electron system again and present the results in Tab. 5. Here we proceed in accordance with the improvements applied to the grid and the Coulomb potential in the one-dimensional system. As a start, we use a calculation (line 28) in which two technical changes are being made compared to the original data $^{18}$ in line 27 to obtain a more accurate $\Gamma$ on the same grid. One is the use of a more accurate integrator, that requires, however, a longer CPU time, the other is a more accurate definition of the harmonic oscillator DVR. Originally it had been defined on a fixed interval on which the parabolic potential had been interpolated along the DVR points. ${ }^{18}$ Here, we define this parabolic potential through its analytical form. For such a representation three DVR points would theoretically be enough. However, it appears that five are needed for numerical stability. The gain in CPU time by nearly a factor of six upon reduction of the harmonic oscillator DVR can be deduced from lines 29 and 30 for the $z$ grid of 140 sine DVRs. In the last lines (31-33) the 185_5_5 grid has been used along with the previously established parametrization for the fitted Coulomb potential $\left(v c u t=10.30 \mathrm{meV}\right.$ and $m_{\kappa}$ 
reduction) at good accuracy and shorter CPU times.

Due to favorable timings, a high accuracy, and a feasible utilization in two-dimensional, quasi two-dimensional, and three-dimensional calculations, we made $v_{\text {cut }}=10.30 \mathrm{meV}$ and $m_{\kappa}=4300$ the standard for all calculations in this work (line 33). The speedup of propagation calculations compared to our old standard (line 27) is by a factor of about 20, i.e. by more than one order of magnitude. Note, that POTFIT itself is faster by a factor of 300, the calculation of the initial state by a factor of 150 . 


\section{References}

1. Cederbaum, L. S., Zobeley, J., Tarantelli, F., Phys. Rev. Lett. 1997, 79, 4778.

2. Santra, R., Cederbaum, L. S., Phys. Rep. 2002, 368, 1.

3. Hergenhahn, U., J. Electron Spectrosc. Relat. Phenom. 2011, 184, 78.

4. Jahnke, T., J. Phys. B: At., Mol. Opt. Phys. 2015, 48, 082001.

5. Barth, S., Joshi, S., Marburger, S., Ulrich, V., Lindblad, A., Öhrwall, G., Björneholm, O., Hergenhahn, U., J. Chem. Phys. 2005, 122, 241102.

6. Aoto, T., Ito, K., Hikosaka, Y., Shigemasa, E., Penent, F., Lablanquie, P., Phys. Rev. Lett. 2006, 97, 243401.

7. Gokhberg, K., Trofimov, A. B., Sommerfeld, T., Cederbaum, L. S., Europhys. Lett. 2005, 72, 228.

8. Gokhberg, K., Averbukh, V., Cederbaum, L. S., J. Chem. Phys. 2006, 124, 144315.

9. Aziz, E. F., Ottosson, N., Faubel, M., Hertel, I. V., Winter, B., Nature 2008, 455, 89.

10. Jahnke, T., Sann, H., Havermeier, T., Kreidi, K., Stuck, C., Meckel, M., Schffler, M., Neumann, N., Wallauer, R., Voss, S., Czasch, A., Jagutzki, O., Malakzadeh, A., Afaneh, F., Weber, Th., Schmidt-Böcking, H., Dörner, R., Nat. Phys. 2010, 6, 139.

11. Schwartz, C. P., Fatehi, S., Saykally, R. J., Prendergast, D., Phys. Rev. Lett. 2010, 105, 198102.

12. Thürmer, S., Ončák, M., Ottosson, N., Seidel, R., Hergenhahn, U., Bradforth, S. E., Slavíček, P., Winter, B., Nat. Chem. 2013, 5, 590.

13. Unger, I., Hollas, D., Seidel, R., Thürmer, S., Aziz, E. F., Slavíček, P., Winter, B., J. Phys. Chem. B 2015, 119, 10750.

14. Stoychev, S. D., Kuleff, A. I., Cederbaum, L. S., J. Am. Chem. Soc. 2011, $133,6817$. 
15. Harbach, P. H. P., Schneider, M., Faraji, S., Dreuw, A., J. Phys. Chem. Lett. 2013, 4, 943.

16. Gokhberg, K., Kolorenč, P., Kuleff, A. I., Cederbaum, L. S., Nature 2014, 505, 661.

17. Alizadeh, E., Orlando, T. M., Sanche, L., Annu. Rev. Phys. Chem. 2015, 66, 379.

18. Bande, A., Gokhberg, K., Cederbaum, L. S., J. Chem. Phys. 2011, 135, 144112.

19. Bande, A., Pont, F. M., Dolbundalchok, P., Gokhberg, K., Cederbaum, L. S., EPJ Web Conf. 2013, 41, 04031.

20. Bande, A., J. Chem. Phys. 2013, 138, 214104.

21. Rogach, A., Ed.; Semiconductor Nanocrystal Quantum Dots; Springer: Wien, New York, 2008.

22. Rossetti, R., Ellison, J. L., Gibson, J. M., Brus, L. E., J. Chem. Phys. 1984, 80, 4464.

23. Alivisatos, A. P., Science 1996, 271, 933.

24. Alivisatos, A. P., J. Phys. Chem. 1996, 100, 13226.

25. Zunger, A., MRS Bulletin 1998, 23, 35.

26. Meyer, H.-D., Manthe, U., Cederbaum, L. S., Chem. Phys. Lett. 1990, 165, 73.

27. Manthe, U., Meyer, H.-D., Cederbaum, L. S., J. Chem. Phys. 1992, 97, 3199.

28. Beck, M. H., Jäckle, A., Worth, G. A., Meyer, H.-D., Phys. Rep. 2000, 324, 1.

29. Meyer, H.-D., Gatti, F., Worth, G. A., Eds., Multidimensional Quantum Dynamics; Whiley-VCH: Weinheim, 2009.

30. Averbukh, V., Cederbaum, L. S., Phys. Rev. Lett. 2006, 96, 053401.

31. Fasshauer, E., Förstel, M., Pallmann, S., Pernpointner, M., Hergenhahn, U., New J. Phys. 2014, 16, 103026. 
32. Winter, B., Nucl. Instr. Meth. Phys. Res. A 2009, 601, 139.

33. Salfi, J., Roddaro, S., Ercolani, D., Sorba, L., Savelyev, I., Blumin, M., Ruda, H. E., Beltram, F., Semicond. Sci. Technol. 2010, 25, 024007.

34. Roddaro, S., Pescaglini, A., Ercolani, D., Sorba, L., Beltram, F., Nano Lett. 2011, 111, 1695

35. Reed, M. A., Bate, R. T., Bradshaw, K., Duncan, W. M., Frensley, W. R., Lee, J. W., Shih, H. D., J. Vac. Sci. Technol. B 1986, 4, 358.

36. Reed, M., Sci. Am. 1993, 268, 118.

37. Grundmann, M., Stier, O., Bimberg, D., Phys. Rev. B 1995, 52, 11969.

38. Jacak, L., Hawrylak, P., Wójs, A., Quantum Dots; Springer: Berlin, Heidelberg, 1998.

39. Petroff, P., DenBaars, S., Superlattices Microstruct. 1994, 15, 15.

40. Krenner, H. J., Sabathil, M., Clark, E. C., Kress, A., Schuh, D., Bichler, M., Abstreiter, G., Finley, J. J., Phys. Rev. Lett. 2005, 94, 057402.

41. Bastard, G., Wave Mechanics Applied to Semiconductor Heterostructures; Les Editions de Physique: Les Ulis, 1992.

42. Pont, F. M., Bande, A., Cederbaum, L. S., Phys. Rev. B 2013, 88, 241304(R).

43. Bande, A., Pont, F. M., Gokhberg, K., Cederbaum, L. S., EPJ Web Conf. 2015, 84, 07002 .

44. Pont, F. M., Bande, A., Cederbaum, L. S., J. Phys.: Condens. Matter 2016, 28, 075301.

45. Electronic archive: New Semiconductor Materials. Characteristics and Properties, http://www.ioffe.ru/SVA/NSM/Semicond/GaAs/basic.html (accessed April 15, 2016).

46. Sajeev, Y., Moiseyev, N., Phys. Rev. B 2008, 78, 075316.

47. Shikin, V., Nazin, S., Heitmann, D., Demel, T., Phys. Rev. B 1991, 43, 11903. 
48. Tannor, D., Introduction to Quantum Mechanics: A Time-Dependent Perspective; Macmillan Education: London, 2007).

49. Sanguinetti, S., Watanabe, K., Tateno, T., Gurioli, M., Werner, P., Wakaki, M., Koguchi, N., J. Cryst. Growth 2003, 253, 71.

50. Shahzadeh, M., Sabaeian, M., AIP Advances 2014, 4, 067113.

51. Aslan, B., Liu, H. C., Kurkusinski, M., Cheng, S.-J., Hawrylak, P., Appl. Phys. Lett. 2003, 82, 630 .

52. Maimon, S., Finkman, E., Bahir, G., Schacham, S. E., Garcia, J. M., Petroff, P. M., Appl. Phys. Lett. 1998, 73, 2003.

53. Nasr, A. S. A. In Infrared Radiation; Morozhenko, V., Ed.; InTech, 2012.

54. Sauerwald, A., Kümmell, T., Bacher, G., Somers, A., Schwertbergerm, R., Reithmaier, J. P., Forchel, A., Appl. Phys. Lett. 2005, 85, 253112.

55. Wang, L., Rastelli, A., Kiravittaya, S., Benyoucef, M., Schmidt, O. G., Adv. Mater. 2009, 21, 2601.

56. Yin, Y., Alivisatos, A. P., Nature 2005, 437, 664.

57. Jäckle, A., Meyer, H.-D., J. Chem. Phys. 1996, 104, 7974.

58. Jäckle, A., Meyer, H.-D., J. Chem. Phys. 1998, 109, 3772.

59. Peláez, D., Meyer, H.-D., J. Chem. Phys. 2013, 138, 014108. 
Figure 1: Two complementary schematic representations of a vertical double QD. In the figure's center the geometric representation of the QDs as cylindrical objects with widths $2 r_{\perp}$ in the transversal $x$ and $y$ direction and with heights $h_{L, R}$ for the left and right QD in the longitudinal $z$ direction is shown. The corresponding electron confinement potential representations are depicted at the bottom and on the right side of the graph connected by dashed lines. The transversal potential is a harmonic oscillator confinement with frequency $\omega_{\perp}$, the longitudinal one a Gaussian confinement for each QD with width parameter $b_{L, R}$. The ICD process is indicated in the Gaussian wells, where the electrons are represented by points and their concerted motion by thick arrows.

Figure 2: (a) Schematic representation of the weak (left) and strong (right) harmonic oscillator confinement potential in $x$ and $y$ and the respective lowest energy wave function in that coordinate for small and large $\omega_{\perp}$ in comparison to a constant Gaussian confinement in the $z$ coordinate. (b) Illustration of two wide (left panel) and narrow (right panel) vertically arranged QDs with a nominal distance $R$ in the weak and strong confinement regime, respectively. An effective near-maximum electron-electron distance $r_{12}^{e f f}$ is indicated for both cases. Relative energies of the two-electron resonance state $L_{1} R_{0}$ (c) and the two-electron ground state $L_{0} R_{0}(\mathrm{~d})$ with respect to the ionization threshold at $E_{L_{0} C}=0 \mathrm{meV}$ as a function of $2 r_{\perp}$. (e) ICD rate $\Gamma$ of the resonance $L_{1} R_{0}$ state as function of $2 r_{\perp}$.

Figure 3: Decay of the modulus squared autocorrelation function $|a(t)|^{2}$ of the $L_{1} R_{0}$ state on a logarithmic scale as a function of time for the default geometry (solid line) and four different frontier geometries (dashed lines for width and dotted lines for height variation). 
Figure 4: (a) Schematic representation of the weak (left) and strong (right) Gaussian confinement potential in $z$ and the respective $L_{0}$ single-electron wave function for small and large $b_{L}$ in comparison to a constant parabolic confinement in $x$ and $y$. (b) Illustration of two vertically arranged QDs with a nominal distance $R$ for a high (left panel) and a flat (right panel) left QD. An effective near-maximum electron-electron distance $r_{12}^{\text {eff }}$ and the spacing $r$ between the two vertically arranged QDs are indicated in both cases. Relative energies of the two-electron resonance state $L_{1} R_{0}$ (c) and the two-electron ground state $L_{0} R_{0}(\mathrm{~d})$ with respect to the ionization threshold at $E_{L_{0} C}=0 \mathrm{meV}$ as a function of $h_{L}$. (e) ICD rate $\Gamma$ of the resonance $L_{1} R_{0}$ state as function of $h_{L}$. (f) Double logarithmic representation of the ICD rate $\Gamma$ as function of the virtual photon energy $E_{v p h}$ (dashed line). Also shown are results of least-squares fits to a $E_{v p h}^{-n}$ behavior. For $4.44 \mathrm{meV} \leq E_{v p h} \leq 5.02 \mathrm{meV}$ the fitted line shown behaves as $\Gamma \propto E_{v p h}^{-1.69}$, for $5.40 \mathrm{meV} \leq E_{v p h} \leq 5.65$ a.u. as $\Gamma \propto E_{v p h}^{-18.2}$. 


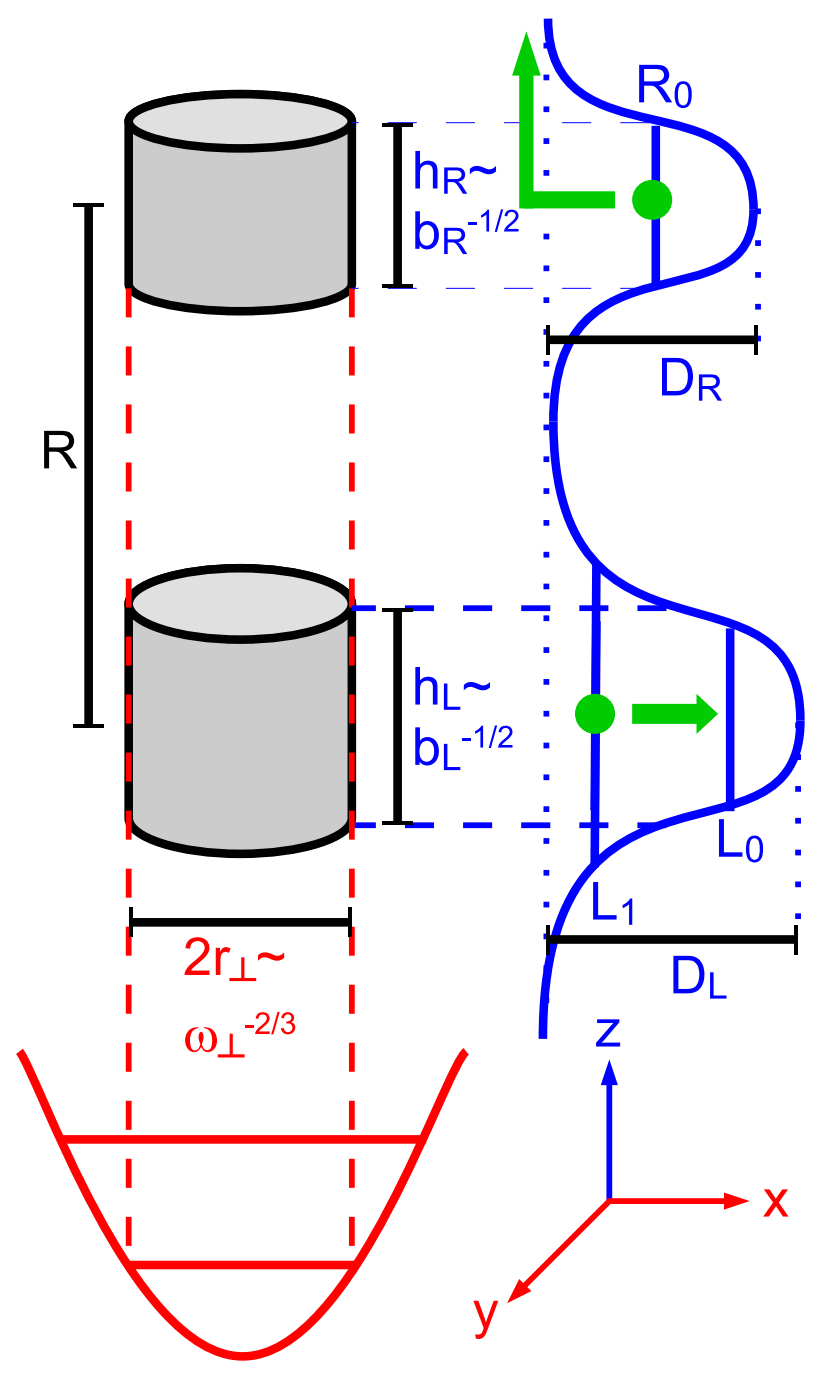

Figure 1

Praphasiri Dolbundalchok, Daniel Peláez, Emad F. Aziz, Annika Bande

J. Comput. Chem. 


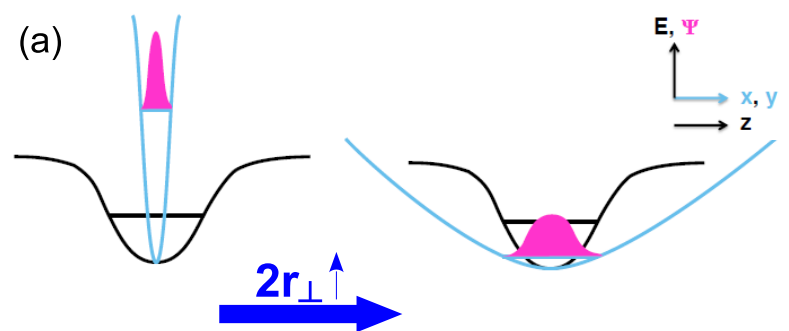

(b)

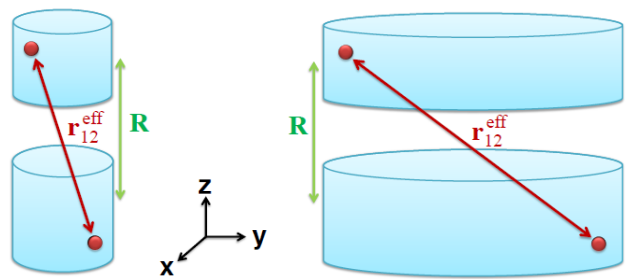

(c)

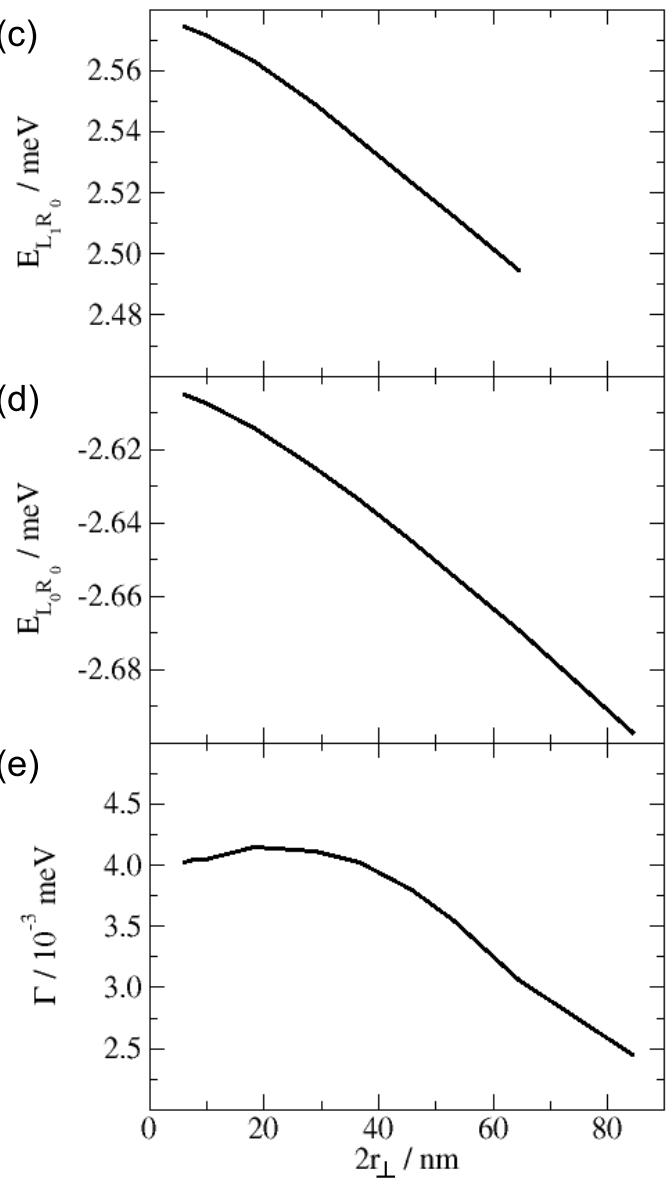


Figure 2

Praphasiri Dolbundalchok, Daniel Peláez, Emad F. Aziz, Annika Bande J. Comput. Chem. 


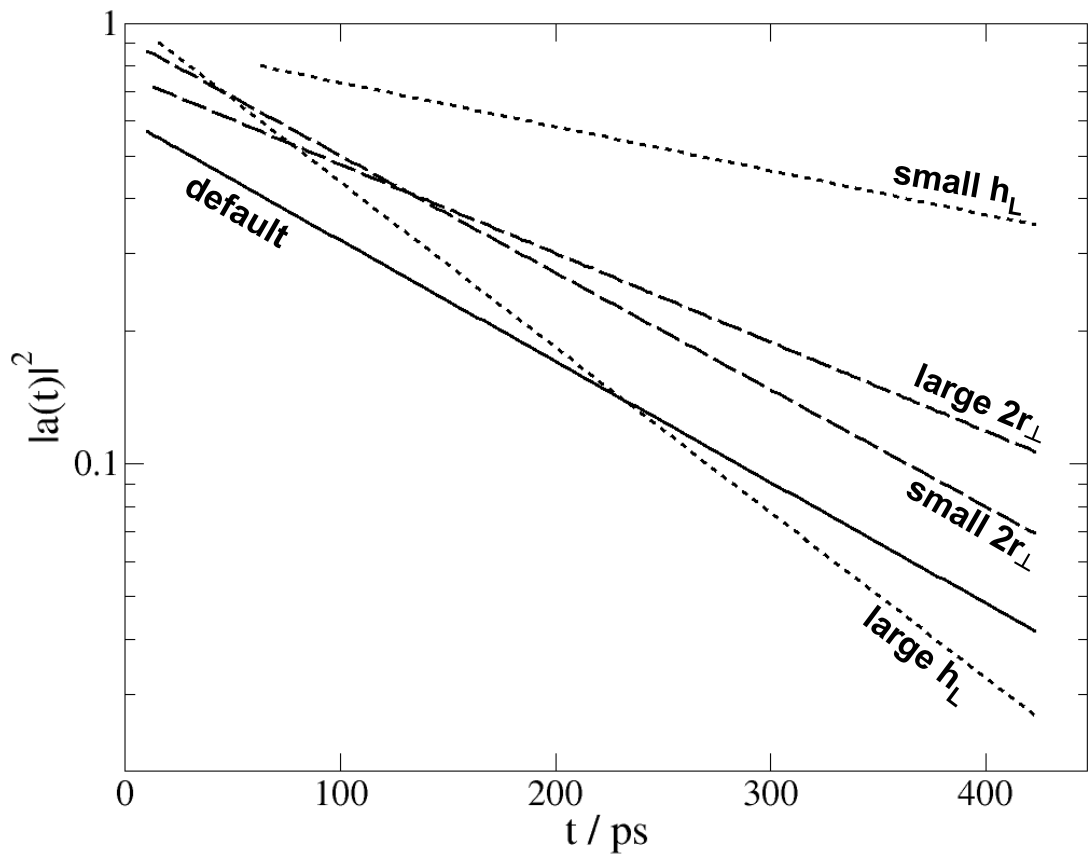

Figure 3

Praphasiri Dolbundalchok, Daniel Peláez, Emad F. Aziz, Annika Bande

J. Comput. Chem. 


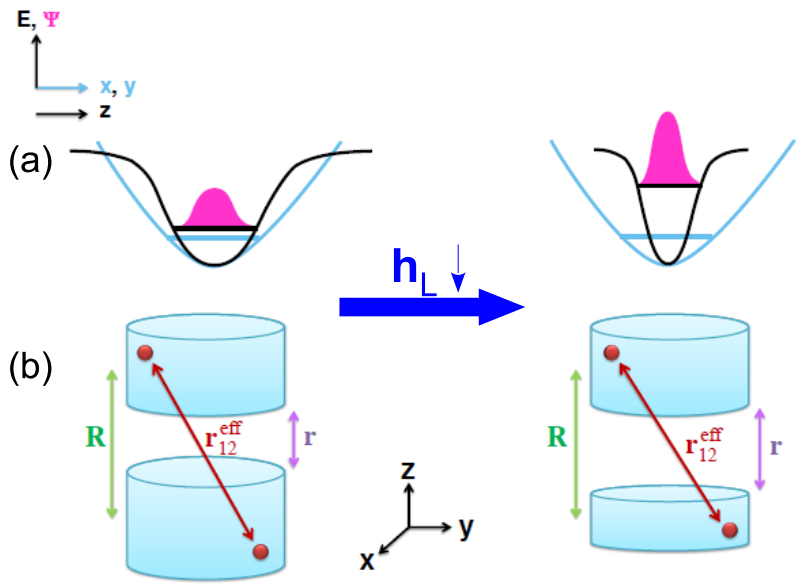

(c)

(d)

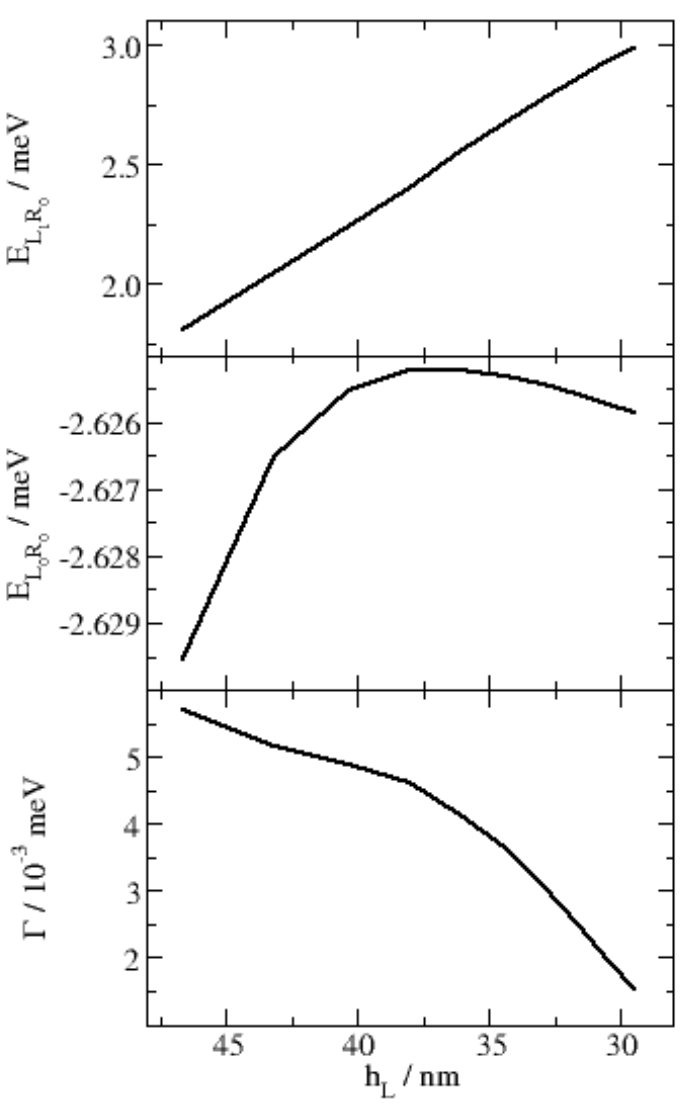

(f)

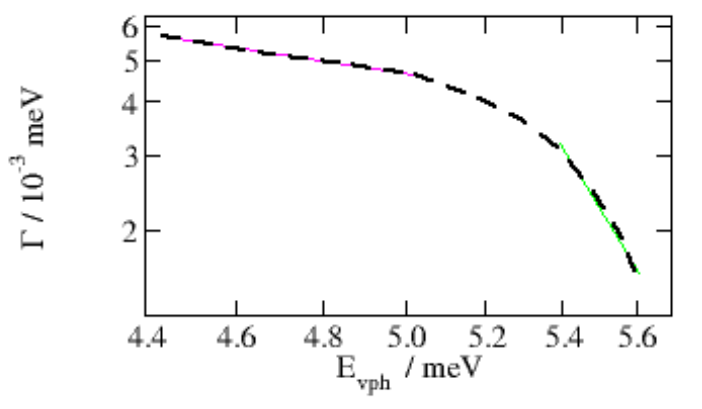


Figure 4

Praphasiri Dolbundalchok, Daniel Peláez, Emad F. Aziz, Annika Bande J. Comput. Chem. 


\begin{tabular}{|c|c|c|c|c|c|}
\hline$\omega_{\perp} /$ a.u. & $\mathbf{2 r}_{\perp} / \mathbf{n m}$ & $\mathbf{E}_{\mathbf{v p h}}$ & $\mathbf{E}_{\mathbf{C}}$ & $\boldsymbol{\Gamma}$ & $\tau / \mathbf{p s}$ \\
\hline 0.2 & 84.32 & 5.160 & 1.286 & 2.451 & 268.54 \\
\hline 0.3 & 64.35 & 5.162 & 1.286 & 3.060 & 215.07 \\
\hline 0.4 & 53.12 & 5.166 & 1.304 & 3.550 & 185.42 \\
\hline 0.5 & 45.78 & 5.169 & 1.315 & 3.798 & 172.39 \\
\hline 0.7 & 36.58 & 5.171 & 1.329 & 4.029 & 163.38 \\
\hline 1.0 & 28.84 & 5.174 & 1.341 & 4.115 & 159.95 \\
\hline 2.0 & 18.17 & 5.189 & 1.355 & 4.144 & 158.84 \\
\hline 5.0 & 9.86 & 5.178 & 1.364 & 4.051 & 162.47 \\
\hline 7.0 & 7.88 & 5.179 & 1.365 & 4.051 & 162.47 \\
\hline 10.0 & 6.21 & 5.180 & 1.367 & 4.028 & 163.41 \\
\hline
\end{tabular}

Table 1: Varying general harmonic oscillator confinement widths $\omega_{\perp}$ and corresponding GaAs quantitites: diameters $2 r_{\perp}$ in $x$ and $y$ direction, virtual photon $E_{v p h}$ and Coulomb energies $E_{C}$ in meV, ICD decay rates $\Gamma / 10^{-3}$ in meV, and decay times $\tau$. 


\begin{tabular}{|c|c|c|c|c|c|c|c|}
\hline $\mathbf{b}_{\mathbf{L}} / \mathbf{a . u .}$ & $\mathbf{h}_{\mathbf{L}} / \mathbf{n m}$ & $\mathbf{E}_{\mathbf{L}_{\mathbf{0}}}$ & $\mathbf{E}_{\mathbf{L}_{\mathbf{1}}}$ & $\mathbf{E}_{\mathbf{v p h}}$ & $\mathbf{E}_{\mathbf{C}}$ & $\boldsymbol{\Gamma}$ & $\tau / \mathbf{p s}$ \\
\hline 0.150 & 46.59 & -7.772 & -3.375 & 4.438 & 1.351 & 5.691 & 115.65 \\
\hline 0.175 & 43.13 & -7.595 & -2.973 & 4.673 & 1.354 & 5.171 & 127.28 \\
\hline 0.200 & 40.34 & -7.433 & -2.616 & 4.862 & 1.345 & 4.878 & 134.92 \\
\hline 0.225 & 38.04 & -7.284 & -2.298 & 5.023 & 1.335 & 4.606 & 142.91 \\
\hline 0.250 & 36.08 & -7.145 & -2.014 & 5.174 & 1.341 & 4.115 & 159.95 \\
\hline 0.275 & 34.41 & -7.016 & -1.760 & 5.295 & 1.337 & 3.646 & 180.52 \\
\hline 0.300 & 32.94 & -6.894 & -1.532 & 5.397 & 1.331 & 3.075 & 214.01 \\
\hline 0.325 & 31.65 & -6.780 & -1.327 & 5.480 & 1.323 & 2.485 & 264.82 \\
\hline 0.350 & 30.50 & -6.671 & -1.142 & 5.557 & 1.324 & 1.941 & 339.01 \\
\hline 0.375 & 29.46 & -6.568 & -0.975 & 5.605 & 1.309 & 1.518 & 433.56 \\
\hline
\end{tabular}

Table 2: Varying general inverse Gaussian confinement widths $b_{L}$ and corresponding GaAs quantitites: heights $h_{L}$ in $z$ direction, energies (in meV) of both one-electron bound states of the QD, $E_{L_{0}}$ and $E_{L_{1}}$, the virtual photon $E_{v p h}$ and the Coulomb interaction $E_{C}$, ICD rates $\Gamma / 10^{-3}$ in meV, and decay times $\tau$ for GaAs QDs are also presented. 


\begin{tabular}{|r|c|c|l|c|c|r|}
\hline line & $\mathbf{N}_{\mathbf{z}}$ & $\mathbf{L}$ & Parameters & $\boldsymbol{\Gamma}$ & $\boldsymbol{\Delta} \boldsymbol{\Gamma}$ & $\mathbf{T}_{\mathbf{P r o p}}$ \\
\hline 1 & 320 & 1084 & $I \leq 10^{-5}$ & 4.4155 & 6.91 & $20 \mathrm{~m}$ \\
\hline 2 & 320 & 1084 & $I=10^{-4}, 8 \mathrm{SPFs}$ & 4.2116 & 1.97 & $28 \mathrm{~m}$ \\
\hline 3 & 320 & 1084 & $I=10^{-6}, 8 \mathrm{SPFs}$ & 4.0932 & -0.90 & $23 \mathrm{~m}$ \\
\hline 4 & 320 & 1084 & $I=10^{-8}, 8 \mathrm{SPFs}$ & 4.1094 & -0.50 & $54 \mathrm{~m}$ \\
\hline 5 & 280 & 1084 & $d_{z}=0.129$ & 4.1121 & -0.44 & $48 \mathrm{~m}$ \\
\hline 6 & 224 & 867 & $d_{z}=0.129, I=10^{-8}, 8 \mathrm{SPFs}$ & 4.1055 & -0.60 & $20 \mathrm{~m}$ \\
\hline 7 & 224 & 867 & $d_{z}=0.129, I=10^{-8}, 10 \mathrm{SPFs}$ & 4.1077 & -0.54 & $56 \mathrm{~m}$ \\
\hline 8 & 224 & 867 & $d_{z}=0.129, I=10^{-6}, 10 \mathrm{SPFs}$ & 4.1049 & -0.61 & $36 \mathrm{~m}$ \\
\hline 9 & 520 & 1625 & $d_{z}=0.160$ & 4.1098 & -0.49 & $145 \mathrm{~m}$ \\
\hline 10 & 400 & 1625 & $d_{z}=0.123$ & 4.1113 & -0.46 & $80 \mathrm{~m}$ \\
\hline 11 & 360 & 1625 & $d_{z}=0.111$ & 4.3899 & 6.29 & $58 \mathrm{~m}$ \\
\hline 12 & 168 & 650 & $d_{z}=0.129, n=2, w=217$ & 4.0937 & -0.88 & $19 \mathrm{~m}$ \\
\hline 13 & 140 & 542 & $d_{z}=0.129, n=2$ & 4.1041 & -0.63 & $11 \mathrm{~m}$ \\
\hline 14 & 112 & 433 & $d_{z}=0.129$ & 4.1427 & 0.30 & $9 \mathrm{~m}$ \\
\hline
\end{tabular}

Table 3: For the effective one-dimensional grid the decay rate $\Gamma / 10^{-3} \mathrm{meV}$, its accuracy $\Delta \Gamma / \%$, and the computation time used for propagations $T_{\text {Prop }}$ in hours $\mathrm{h}$ and minutes $\mathrm{m}$ are displayed for $N_{i}$ grid points in the $i$ th direction, grid lengths $L / \mathrm{nm}$, integrator accuracy $I / \mathrm{meV}$, number of SPFs, grid point density $d_{z}$, CAP order $n$ and widths $w / \mathrm{nm}$. 


\begin{tabular}{|r|c|c|l|c|c|c|}
\hline line & $\mathbf{N}_{\mathbf{z}}$ & $\mathbf{L}$ & Parameters & $\boldsymbol{\Gamma}$ & $\boldsymbol{\Delta} \boldsymbol{\Gamma}$ & $\mathbf{T}_{\text {Prop }}$ \\
\hline 15 & 168 & 650 & $n=2, w=325$ & 4.0994 & -0.75 & $14 \mathrm{~m}$ \\
\hline 16 & 169 & 650 & $n=2, w=325, \mathrm{EXP}$ & 4.1254 & -0.11 & $45 \mathrm{~m}$ \\
\hline 17 & 140 & 542 & $n=3$ & 4.1213 & -0.21 & $16 \mathrm{~m}$ \\
\hline 18 & 140 & 542 & $n=4$ & 4.1094 & -0.50 & $16 \mathrm{~m}$ \\
\hline 19 & 168 & 650 & $n=2, w=433$ & 4.1049 & -0.61 & $14 \mathrm{~m}$ \\
\hline 20 & 140 & 542 & $m_{\kappa}=130$ & 4.1109 & -0.47 & $48 \mathrm{~m}$ \\
\hline 21 & 140 & 542 & $m_{\kappa}=80$ & 3.8196 & -7.52 & $32 \mathrm{~m}$ \\
\hline 22 & 140 & 542 & $m_{\kappa}=N_{z}$, vcut $=10.30$ & 4.0997 & -0.74 & $32 \mathrm{~m}$ \\
\hline 23 & 140 & 542 & $m_{\kappa}=N_{z}$, vcut $=5.16$ & 4.4069 & 6.70 & $32 \mathrm{~m}$ \\
\hline 24 & 185 & 542 & $m_{\kappa}=185$ & 4.1030 & -0.66 & $20 \mathrm{~m}$ \\
\hline 25 & 185 & 542 & $m_{\kappa}=172$ & 4.1016 & -0.69 & $20 \mathrm{~m}$ \\
\hline 26 & 185 & 542 & $m_{\kappa}=127$ & 4.0979 & -0.78 & $11 \mathrm{~m}$ \\
\hline
\end{tabular}

Table 4: For the effective one-dimensional grid the decay rate $\Gamma / 10^{-3} \mathrm{meV}$, its accuracy $\Delta \Gamma / \%$, and the computation time used for propagations $T_{\text {Prop }}$ in hours $\mathrm{h}$ and minutes $\mathrm{m}$ are displayed for $N_{i}$ grid points in the $i$ th direction, grid lengths $L / \mathrm{nm}$, exponential DVR (EXP), CAP order $n$ and widths $w / \mathrm{nm}$, number of SPPs $m_{\kappa}$, and cutoff $v c u t / \mathrm{meV}$ for Coulomb potential. 


\begin{tabular}{|r|c|c|c|l|c|c|r|}
\hline line & $\mathbf{N}_{\mathbf{z}}$ & $\mathbf{L}$ & $\mathbf{N}_{\mathbf{x}, \mathbf{y}}$ & Parameters & $\boldsymbol{\Gamma}$ & $\boldsymbol{\Delta} \boldsymbol{\Gamma}$ & $\mathbf{T}_{\text {Prop }}$ \\
\hline 27 & 320 & 1084 & $8^{a}$ & previous $^{18} I \leq 10^{-5}$ & 4.2859 & 3.77 & $2119 \mathrm{~h}$ \\
\hline 28 & 320 & 1084 & 8 & $I=10^{-6}$ & 4.1000 & -0.73 & $3096 \mathrm{~h}$ \\
\hline 29 & 140 & 542 & 8 & & 4.1055 & -0.60 & $427 \mathrm{~h}$ \\
\hline 30 & 140 & 542 & 5 & & 4.1005 & -0.72 & $75 \mathrm{~h}$ \\
\hline 31 & 185 & 542 & 5 & & 4.0843 & -1.11 & $134 \mathrm{~h}$ \\
\hline 32 & 185 & 542 & 5 & vcut $^{-6} 10.30, m_{\kappa}=4300$ & 4.1302 & 0.00 & $100 \mathrm{~h}$ \\
\hline 33 & 185 & 542 & 5 & vcut $=10.30, m_{\kappa}=1181$ & 4.1640 & 0.82 & $39 \mathrm{~h}$ \\
\hline
\end{tabular}

${ }^{a}$ This harmonic oscillator DVR was defined on eight grid points by interpolation.

Table 5: For the quasi one-dimensional grid the decay rate $\Gamma / 10^{-3} \mathrm{meV}$, its accuracy $\Delta \Gamma$, and the computation time used for propagations $T_{\text {Prop }}$ in hours $\mathrm{h}$ and minutes $\mathrm{m}$ are displayed for $N_{i}$ grid points in the $i$ th direction, grid lengths $L / \mathrm{nm}$, integrator accuracy $I / \mathrm{meV}$, number of SPPs $m_{\kappa}$, and cutoff $v c u t / \mathrm{meV}$ for Coulomb potential. 